\title{
Tracking the Position and Rotational Orientation of a Catheter Using a Transmit Array System
}

\author{
Haydar Celik*, Member, IEEE, Davut I. Mahcicek, OytunK. Senel, Graham A. Wright, Member, IEEE, and \\ Ergin Atalar, Member, IEEE
}

\begin{abstract}
A new method for detecting the rotational orientation and tracking the position of an inductively coupled radio frequency (ICRF) coil using a transmit array system is proposed. The method employs a conventional body birdcage coil, but the quadrature hybrid is eliminated so that the two excitation channels can be used separately. The transmit array system provides RF excitations such that the body birdcage coil creates linearly polarized and changing RF pulses instead of a conventional rotational forward-polarized excitation. The receive coils and their operations are not modified. Inductively coupled RF coils are constructed on catheters for detecting rotational orientation and for tracking purposes. Signals from the anatomy and from tissue close to the ICRF coil are different due to the new RF excitation scheme: the ICRF coil can be separated from the anatomy in real time, and after doing so, a color-coded image is reconstructed. More importantly, this novel method enables a real-time calculation of the absolute rotational orientation of an ICRF coil constructed on a catheter.

Modified FLASH and TrueFISP sequences are used for the experiments. The acquired images from this technique show the feasibility of different applications, such as catheter tracking. Furthermore, applications where knowledge of the rotational orientation of the catheter is important, such as magnetic resonance-guided endoluminal-focused ultrasound, RF ablation, side-looking optical imaging, and catheters with side ports for needles, become feasible with this method.
\end{abstract}

Index Terms-Catheter tracking, inductively coupled radio-frequency (ICRF) coil, interventional MRI, rotational orientation, transmit array.

\section{INTRODUCTION}

$\mathbf{L}$ OCATING interventional devices quickly and precisely is necessary to perform minimally invasive operations. Generally, X-rays are used for these operations because of their high-resolution instrument visualization, however, X-rays cannot provide soft tissue contrast and they expose patients and staff to ionizing radiation. Magnetic resonance imaging

Manuscript received November 18, 2012; revised January 29, 2013; accepted January 30, 2013. Date of publication February 13, 2013; date of current version March 29, 2013. Asterisk indicates corresponding author.

${ }^{*} \mathrm{H}$. Celik was with the Electrical and Electronics Engineering Department, Bilkent University, TR-06800 Ankara, Turkey. He is now with the Sunnybrook Research Institute, Toronto, ON, M4N 3M5 Canada (e-mail: haydari@gmail. com).

D. I. Mahcicek and E. Atalar are with the Electrical and Electronics Engineering Department, Bilkent University, TR-06800 Ankara, Turkey.

O. K. Senel is with the Faculty of Veterinary Medicine, Ankara University, TR-06800 Ankara, Turkey.

G. A. Wright is with the Sunnybrook Research Institute, Toronto, ON, M4N 3M5 Canada.

Color versions of one or more of the figures in this paper are available online at http://ieeexplore.ieee.org.

Digital Object Identifier 10.1109/TMI.2013.2247047
(MRI) offers impressive soft tissue contrast is a nonionizing imaging modality, but it cannot detect and track interventional devices such as catheters, guidewires, and biopsy needles in real time. Various techniques have been developed for identifying interventional devices for such operations. Active tracking methods use antennae [1] or small coils [2] connected as separate receive channels. Passive techniques use contrast materials or metallic susceptibility for visualization, so there is no connection to the MR scanner [3]-[5]. A third method can be defined as wireless active visualization [6], [7], using inductively coupled radio-frequency (ICRF) coils. In this blended method, the tracking ICRF coil is coupled to a surface receive coil inductively but there is no wired connection to the MR scanner. Although passive and active catheter-tracking methods have distinct advantages, passive tracking methods have reliability problems, and active tracking techniques pose device design and safety concerns [2], [8]-[16].

Due to frictional forces and catheter elasticity, applied torque may not translate to the tip of the catheter; therefore its rotational orientation may be unknown even when the torque is controlled by skilled cardiologists. Determining the rotational orientation of the cylindrically symmetric catheters is difficult, but this information may be useful when the asymmetric catheter design is necessary, such as in MR-guided intravascular-focused ultrasound [17], [18] with independent transducer arrays [17]-[19] and radio-frequency ablation. Although some researchers have introduced solutions for the determining a catheter's rotational orientation [20], [21], this is a relatively untouched subject.

Transmit array systems have recently been introduced for homogenizing high-frequency $B_{1}$ fields [22]-[25], but their capabilities are also used for catheter tracking by inducing reverse polarization [23]. In the current study, a new method that uses a transmit array system is presented to 1) detect rotational orientation and 2) track the position of the interventional devices on which ICRF coils are constructed [7], [26]-[29]. Two mechanisms are used in the transmit and receive chains; the first is the coupling of the transmit body birdcage coil to the ICRF coil; the second is the coupling of modulated spins around the ICRF coil to a receive phased array coil. Conventional RF excitation pulses are modified such that linearly polarized $B_{1}$ fields with changing direction are created during the transmit phase instead of a forward-polarized magnetic field. As a result, the anatomy image shifts in the phase-encoding direction so that two copies of an ICRF coil image are created: one shifts in the phase-encoding direction and the other shifts in the opposite direction. Moreover, the ICRF coil images have a phase difference directly 
proportional to the rotational orientation of the ICRF coil. In this way, the method enables simultaneous acquisition of anatomy and ICRF coil images with rotational orientation information.

\section{THEORY}

Modulated RF excitations are used in saturation bands for eliminating artifacts [30], and for off-center slice excitation [31]. However, such changes are limited to the phase modulations of conventional forward-polarized RF excitations. Understanding the results of phase and/or amplitude modulations of linearly polarized RF excitations requires rigorous analysis (see the Appendix).

\section{A. General Theory of Circulating RF Excitations}

1) Conventional Body Coil Excitation: A body birdcage coil has two orthogonal transmit channels. If only one transmit channel is excited, a linearly polarized magnetic field is created in either the $x$ or $y$ direction. Conventionally, a quadrature hybrid is used to deliver the excitation signal through a single channel [32], [33] [Fig. 1(a)]. The quadrature hybrid divides the signal into two signals with equal magnitude and a $90^{\circ}$ phase difference, which generates a forward-polarized field [32]. This rotational excitation is two times more efficient in total body specific absorption rate (SAR) and four times better in peak SAR than a linear excitation [30].

2) Changing RF Fields: In this study, a transmit array system is used to deliver modified RF excitations to two channels of a body birdcage coil in place of the quadrature hybrid. By modifying the conventional sequences a linearly polarized RF excitation can be obtained instead of a forward-polarized one.

In conventional MR imaging, the RF excitation scheme is constant throughout the sequence. Although it is not easy to determine the results of arbitrary modifications of RF excitations, the effects of periodic changes can be calculated using the Fourier theorem. Fig. 1(b)-(e) shows a modified linearly polarized RF excitation scheme in which RF amplitudes and phases are changed at each repetition time (TR). Four different magnetic fields are repeated periodically, such that $B_{p}=B_{p+4}$, where $p$ is the index of the TR. Changing the direction of the linear polarization causes the excitation vector to circulate in a counter-clockwise direction. At the first TR, the RF channel on the $\mathrm{x}$-axis $\left(\mathrm{RF}_{1}\right)$ is on and the channel along the $y$-axis $\left(\mathrm{RF}_{2}\right)$ is off. As a result, the direction of the linear excitation field is on the $\mathrm{x}$-axis. The second RF channel is on at the next TR and the vector is on the $y$-axis with a relative phase of $\pi / 2$. At the third $T R, R F_{1}$ is on but with a relative phase of $\pi$. Last, at the fourth TR, RF is on the $-y$ direction with a phase of $3 \pi / 2$. In this periodic scheme, the $p$ th magnetic field can be shown as $B_{p}=B e^{i p \pi / 2}$, where $B$ is the reference-complex magnetic field expression. Assuming linear increments in the k-space position with each TR and using the Fourier theorem [34], the resulting phase modulation in k-space produces a shift in the image domain. Specifically, the modulation causes a $N_{y} / P$ pixel shift in the image domain, where $N_{y}$ is the number of phase-encoding lines and $P$ is the period of the changing excitation, which equals 4 in the above case (Fig. 1).

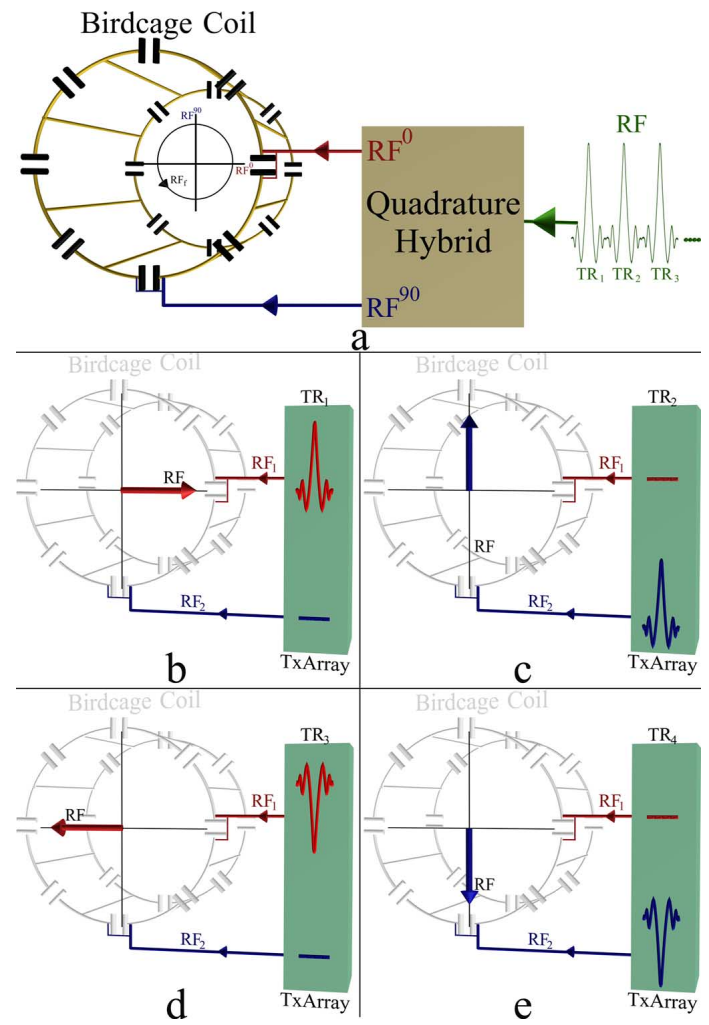

Fig. 1. Standard and modified RF excitation schemes of the transmit body birdcage coil. a: The quadrature hybrid divides the input excitation signal into halves with a $90^{\circ}$ phase difference and enables a forward-polarized field inside a birdcage coil. b: Channel excitations change such that a linearly polarized field with a circulating polarization vector is created. The RF is on the $x$-axis in the first repetition time (TR), because $R_{1}$ is on and $R F_{2}$ is off. c: In the second TR, the RF is on the $y$-axis. The RF is on the $x$-axis but in the negative direction in the third TR (d) and in the fourth TR, the RF is on the negative $y$-direction (e) This scheme is periodic with period $P$ and in this example $P=4$.

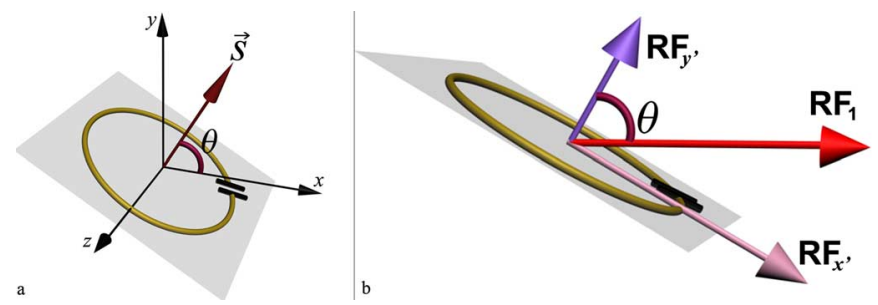

Fig. 2. a: An ICRF coil and its orientation, $\theta$. b: Interaction of the linearly polarized RF and the ICRF coil

\section{B. Effect of Changing RF Fields on the ICRF Coil}

With the excitation, the ICRF coil is exposed to a different coupling mechanism than spins in the tissue are. The magnetic field transmitted by the body coil induces a current in the ICRF coil, which introduces a secondary field. When the excitation field is linear, coupling of the transmit field and the ICRF coil is directly related to the coil's orientation. Fig. 2 shows the relation of the ICRF coil orientation and the excitation vector. As an example, assume $R_{1}$ is applied and the ICRF coil is tilted such that the surface normal vector of the ICRF coil makes a $\theta$ angle with $\mathrm{RF}_{1}$. This excitation pulse can be decomposed into two orthogonal fields, $\mathrm{RF}_{x^{\prime}}$ and $\mathrm{RF}_{y^{\prime}}$, which are perpendicular and parallel components to the surface normal vector of the 
ICRF coil, respectively. By using Faraday's law of induction, one can conclude that $\mathrm{RF}_{y^{\prime}}$ induces a current on the ICRF coil but $\mathrm{RF}_{x^{\prime}}$ does not. Therefore, the effective magnetic field component is $B \cos \theta$ and the secondary field produced by the ICRF coil becomes

$$
B_{\mathrm{ICRF}, 1}=B C \cos \theta
$$

where $C$ is a complex amplification factor due to the ICRF coil.

If one applies this operation to the other components of the RF excitation pulses shown in Fig. 1, the magnetic fields due to the excitation profile become $B_{\mathrm{ICRF}, 2}=B C \sin \theta, B_{I C R F, 3}=$ $-B C \cos \theta$, and $B_{\mathrm{ICRF}, 4}=-B C \sin \theta$. This excitation profile is periodic, but not as simple as the previous case because there is now phase and amplitude modulation. A simpler form can be obtained by decomposing each excitation

$$
\begin{aligned}
& B_{\mathrm{ICRF}, 1}=B C \cos \theta=B C \alpha+B C \beta \\
& B_{\mathrm{ICRF}, 2}=B C \sin \theta=B C \alpha e^{i \pi / 2}+B C \beta e^{i 3 \pi / 2} \\
& B_{\mathrm{ICRF}, 3}=-B C \cos \theta=B C \alpha e^{i \pi}+B C \beta e^{i \pi} \\
& B_{\mathrm{ICRF}, 4}=-B C \sin \theta=B C \alpha e^{i 3 \pi / 2}+B C \beta e^{i \pi / 2} .
\end{aligned}
$$

Above, $\alpha=(\cos \theta+i \sin \theta) / 2$ and $\beta=(\cos \theta-i \sin \theta) / 2$. One can express the $p$ th excitation in a sequence as $B_{p}^{*}=$ $B C \alpha e^{i(p-1) \pi / 2}+B C \beta e^{-i(p-1) \pi / 2}$. Therefore, the resultant image consists of two copies of the original ICRF coil image. The first image shifts in the phase-encoding direction in the same way that the anatomy image shifts. The second image is called a "ghost" and shifts in the opposite direction, away from the anatomy, which enables tracking of the ICRF coil. Furthermore, the phase of the ratio between signals in the two images equals two times the ICRF coil orientation, $2 \theta$, which enables calculating the rotational orientation of the ICRF coil simultaneously. A more-detailed analysis is provided in the Appendix.

\section{METHODS}

In the present study, the aims are 1) to separate images of the anatomy and interventional device and 2) to find the rotational orientation of the device using a linearly polarized body birdcage coil and an ICRF coil on the device. The identical RF pulse shape with changing amplitude and phase is used to excite the body birdcage coil, as described in Fig. 1. The quadrature hybrid excitation pattern on the body coil is replaced with a two-channel linear transmit pattern. The FLASH and TrueFISP sequences (see Section III-A below) are modified and a linearly polarized field, modulated as a function of the k-space position, is delivered to the body coil using this transmit system. To avoid wrapping because of the shift, the field-of-view (FOV) was doubled in all experiments.

In this work, 3T Siemens TIMTrio (Siemens AG, Erlangen, Germany) imaging and two channels of an eight-channel transmit array system were used. Siemens phased-array receive coils (body and spine matrix) were used for reception. A MATLAB (version 7.6; Mathworks Inc., Natick, MA, USA) code was written to reconstruct and calculate the rotational orientation and color-code the ghost for catheter tracking.

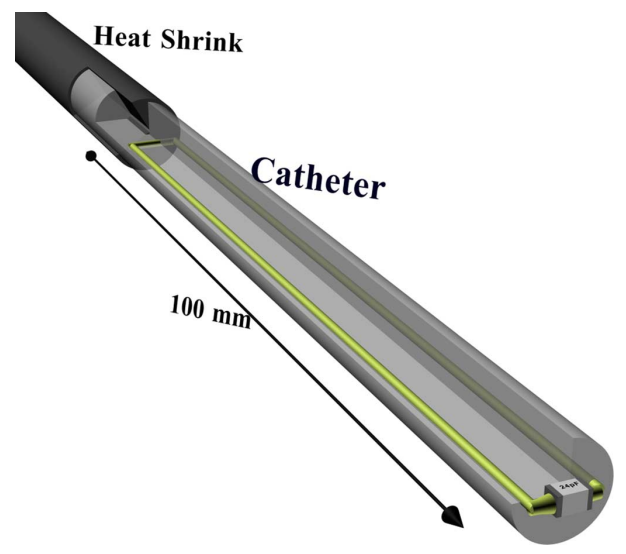

Fig. 3. ICRF coil is a loop of magnet wire resonated by a tuning capacitor. 100 -mm-long ICRF coil was constructed on a $6 \mathrm{~F}$ catheter.

\section{A. Sequences}

The presented technique was applied to two fast and clinically relevant sequences, FLASH and TrueFISP. A MATLAB simulation for the TrueFISP sequence was used to show that the shifting mechanism is still valid for larger flip angles; however, the TrueFISP sequence is sensitive to field inhomogeneities. To understand signal behavior for the anatomy and the ICRF coil, computer simulations were conducted using MATLAB. The anatomy and catheter signals were simulated using the following parameters: $T_{1}=200 \mathrm{~ms}, T_{2}=60 \mathrm{~ms}, \mathrm{TR}=5.8 \mathrm{~ms}$, $\mathrm{TE}=2.9 \mathrm{~ms}$, and flip angle $=50^{\circ}$.

\section{B. Rotational Orientation}

Two ICRF coils with different diameters but the same length were constructed for the rotational orientation experiments and rotated approximately $5^{\circ}$ in each step (Fig. 3). The first ICRF was $100 \mathrm{~mm}$ long and constructed on a Teflon catheter with an outer diameter of $2 \mathrm{~mm}$ (6 French, F) using coated copper wire $0.4 \mathrm{~mm}$ in diameter. A heat shrink tube was used for isolation, resulting in a prototype device with an outer diameter of $3 \mathrm{~mm}(9 \mathrm{~F})$. It was tuned by a $24 \mathrm{pF}$ ceramic chip capacitor (ATC, Huntington Station, NY, USA) to $123.23 \mathrm{MHz}$ using an HP 8753D network analyzer (Agilent Technologies, Santa Clara, CA, USA). The second ICRF coil was the same length with a $22 \mathrm{~F}$ total diameter. Both ICRF coils were placed on the $z=0$ plane with $22^{\circ}$ angle to the $y=0$ plane.

To determine the ICRF coil's rotational orientation, which is two times the phase difference between the ICRF coil and ghost images, a magnitude squared weighting of accumulating complex exponentials method [35] was used. For the $P=4$ case explained in the theory section, the center of the final image was the border of the original shifted images (Fig. 4). Cutting one side and multiplying with the conjugate of the other side of the original images provided appropriate weighting of the phase information, which is two times the absolute orientation of the ICRF coil. For proof of principle, a FLASH experiment simulation was performed to change the rotational orientation of the ICRF coil.

A plastic container (dimensions $15 \mathrm{~cm} \times 10 \mathrm{~cm} \times 30 \mathrm{~cm}$ ) filled with vegetable oil was used as a phantom and a protractor 


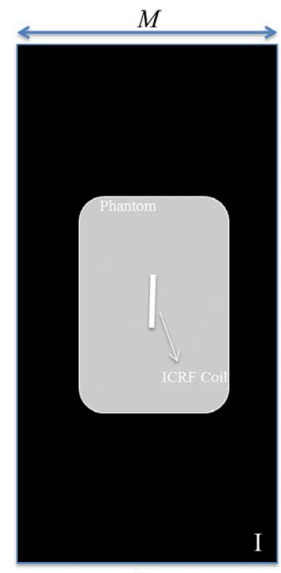

a

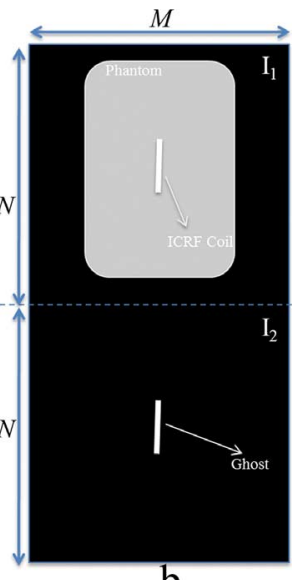

b
Fig. 4. Calculation of weighted exponentials. If a phantom is at the center of image (a), changing the RF excitation causes shifting of the phantom and ICRF coil image in one direction and the ghost in the opposite direction. Rotational orientation is calculated using the shifted images.
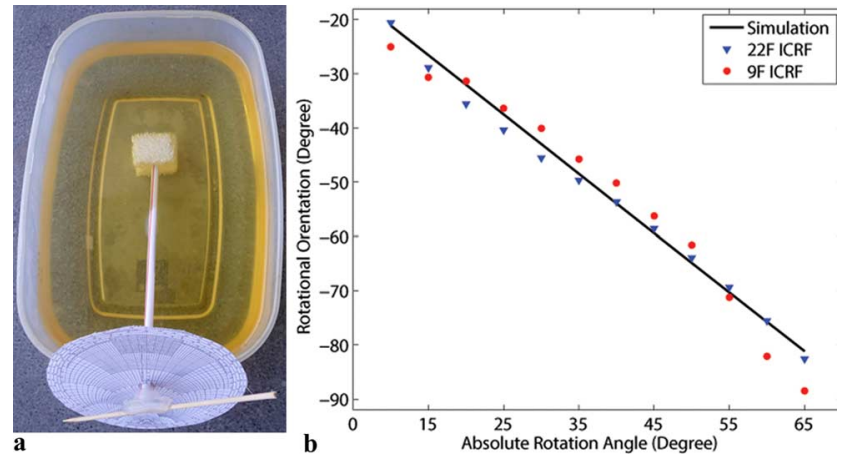

Fig. 5. Oil phantom setup and rotational orientation experiment result. a: A protractor was used to achieve equally spaced angles. b: The $9 \mathrm{~F}$ and $22 \mathrm{~F}$ ICRF coils showed a very impressive correlation with the MATLAB FLASH simulation.

was used to change the rotational angle [Fig. 5(a)]. Before each acquisition, the ICRF coils were rotated equally.

\section{Catheter Tracking}

The first ICRF coil, 9F, was also used in the phantom and animal experiments and was similar to the one in the author's previous study [32]. The same oil phantom box as above was used and four $\mathrm{KCl}$-solution-filled straws were inserted.

The FLASH sequence was modified to obtain circulating polarizations with the following parameters: $\mathrm{TR}=9.8 \mathrm{~ms}$, $\mathrm{TE}=4.1 \mathrm{~ms}$, slice thickness $5 \mathrm{~mm}$, flip angle $30^{\circ}$, FOV 300 $\mathrm{mm} \times 300 \mathrm{~mm}$, and imaging matrix $256 \times 256$. Proof-of-principle experiments were conducted with a modified TrueFISP sequence using the following parameters: $\mathrm{TR}=5.8 \mathrm{~ms}$, $\mathrm{TE}=2.9 \mathrm{~ms}$, slice thickness $=5 \mathrm{~mm}$, flip angle $=50^{\circ}, \mathrm{FOV}$ $300 \mathrm{~mm} \times 300 \mathrm{~mm}$, and imaging matrix $256 \times 256$.

\section{Transmit Array Calibration}

Two channels of the transmit array system were used in the site. After each system boot, different phase offsets between the channels were observed. Therefore, before each experiment, the phase difference was calibrated using the reverse-polarized mode of the birdcage coil [23], [32].

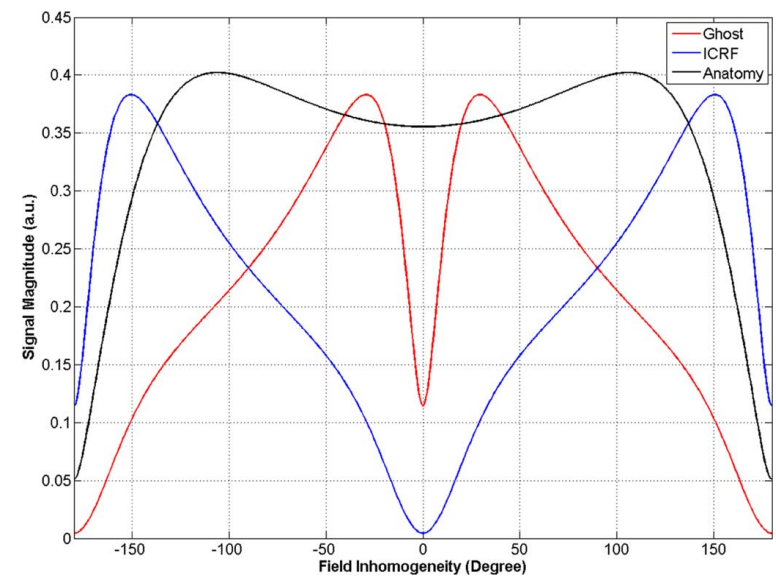

Fig. 6. Simulation result for TrueFISP. This plot shows the effect of field inhomogeneity on the ICRF coil and the ghost images. The anatomy signal was not affected, but there was a $180^{\circ}$ phase difference between the ICRF coil and the ghost.

\section{E. Animal Imaging Experiments}

The animal imaging experiments were approved by the Bilkent University ethics committee, Ankara, Turkey. Five $\mathrm{mg} / \mathrm{kg}$ of ketamine and $40 \mathrm{mg} / \mathrm{kg}$ of xylazine were injected into a New Zealand rabbit to induce general anesthesia. Then the 9F ICRF coil was lubricated and inserted via the mouth into the duodenum, as described in our previous study [32]. Images were acquired using the following FLASH sequence: $\mathrm{TR}=9.8 \mathrm{~ms}, \mathrm{TE}=4.1 \mathrm{~ms}$, slice thickness $=5 \mathrm{~mm}$, flip angle $=30^{\circ}$, FOV $300 \mathrm{~mm} \times 300 \mathrm{~mm}$, and imaging matrix $256 \times 256$.

\section{RESULTS}

\section{A. TrueFISP Simulation}

Fig. 6 presents the simulation result and shows the anatomy, ICRF coil, and ghost signals. As a function of field inhomogeneity, these signals differ significantly. The anatomy signal does not change and shows the TrueFISP characteristics: it has a large plateau between $-120^{\circ}$ and $120^{\circ}$ following a sharp signal drop, which causes inhomogeneity artifacts on the TrueFISP images. On the other hand, signal variations of the ICRF coil and the ghost are significant because of the orientation dependence of the ICRF coil signal. The ICRF coil has two dips: the first one is at the center (on resonance) and drops close to zero, and the second is at $180^{\circ}$. The ICRF coil and its ghost have the same shape with respect to field inhomogeneity, but there is a $180^{\circ}$ phase difference. This behavior introduces another parameter for the method; field inhomogeneity can change the amplitudes of the ICRF coil and its ghost. For example, the ICRF coil signal has a dip at the center while the ghost signal is nonzero, so the center can be used to suppress the ICRF coil signal. The signal variations were confirmed by phantom experiments, but because of the space limitations these images are not given in a figure. Note that signal levels of the ICRF coil and the ghost were normalized and amplification factors were not taken into account. 


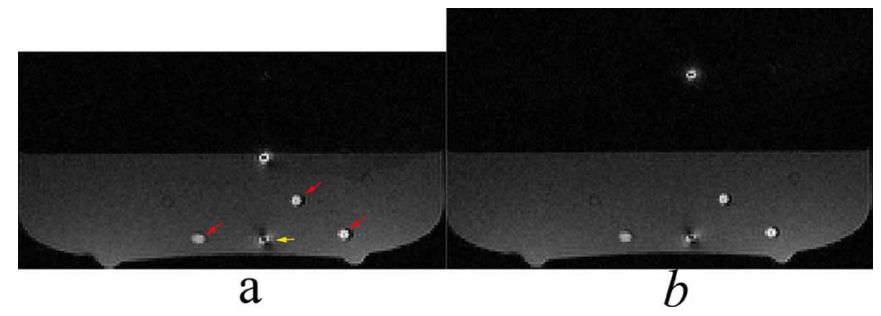

Fig. 7. Transversal images with two different periods of the changing excitation, $P$, and the phase-encoding lines of $N_{y}=256$. The images show that the number of shifting pixels can be adjusted. Three straws are filled with $\mathrm{KCl}$ solution (red arrows) and one straw has the ICRF coil inside it (yellow arrow). (a) When $P=16$, there are only 32 pixels between the ICRF coil and the ghost (no arrow). (b) When $P=8$, there are 64 pixels between the ICRF coil and the ghost. Note that the images have been cropped.

\section{B. Rotational Orientation of the ICRF Coil}

Fig. 5 shows the rotational orientation experiment setup and the results obtained using two different ICRF coils. In each measurement, one of the ICRF coils was rotated one step, which is approximately equal to $5^{\circ}$. The experiment was repeated for both ICRF coils. Considering possible experimental setup errors, the standard deviation was calculated to be $4.0^{\circ}$ and $1.7^{\circ}$ for the $9 \mathrm{~F}$ and $22 \mathrm{~F}$ catheters, respectively. Although smaller ICRF coils have a lower signal quality and signal amplification, the results for both catheters are similar and successful [Fig. 5(b)].

\section{Tracking the ICRF Coil}

In the theory section, the period of the changing excitation, $P$, was assumed as an example to be 4 . The pixel shift can be adjusted by changing the period. $P$ values were 16 and 8 in Fig. 7(a) and (b), respectively. Three straws were filled with $\mathrm{KCl}$ solution (red arrows) and one straw had the ICRF coil (yellow arrow) in it; $N_{y}=256$ and a modified FLASH sequence was used. Note that KCl-filled straws have higher signals than the ICRF coil.

Fig. 8 shows transversal phantom images with counter-clockwise circulation [Fig. 8(a)], clockwise circulation [Fig. 8(b)], and an A-P phase-encoding direction [Fig. 8(c)].

Fig. 9 shows different imaging planes with the FLASH sequence [coronal (a), sagittal (b), and oblique (c)] and the TrueFISP sequence [transversal (d), oblique (e), and coronal (f) images] when $P=4$. Although the signal levels of the straws are higher than those of the ICRF coil, the method can successfully separate the ghost from the phantom. As a result, the ghost's signal-to-noise ratio (SNR) is very high, as evident in the performance plots. The main drawback of the TrueFISP sequence is field inhomogeneity at 3T [Fig. 9(f)]. The TrueFISP sequence is more sensitive to off-resonance effects, requiring higher-order shimming for good image quality.

\section{Animal Experiment}

Fig. 10 shows the rabbit experiment results with the FLASH sequence on the sagittal and oblique planes. The performance plots show that signal level of the ghost is higher than the noise that enables color-coded images. The ICRF coil was successfully separated from the anatomy on the sagittal [Fig. 10(a)] and oblique [Fig. 10(b)] planes alike. Fig. 10(b) shows that the re-
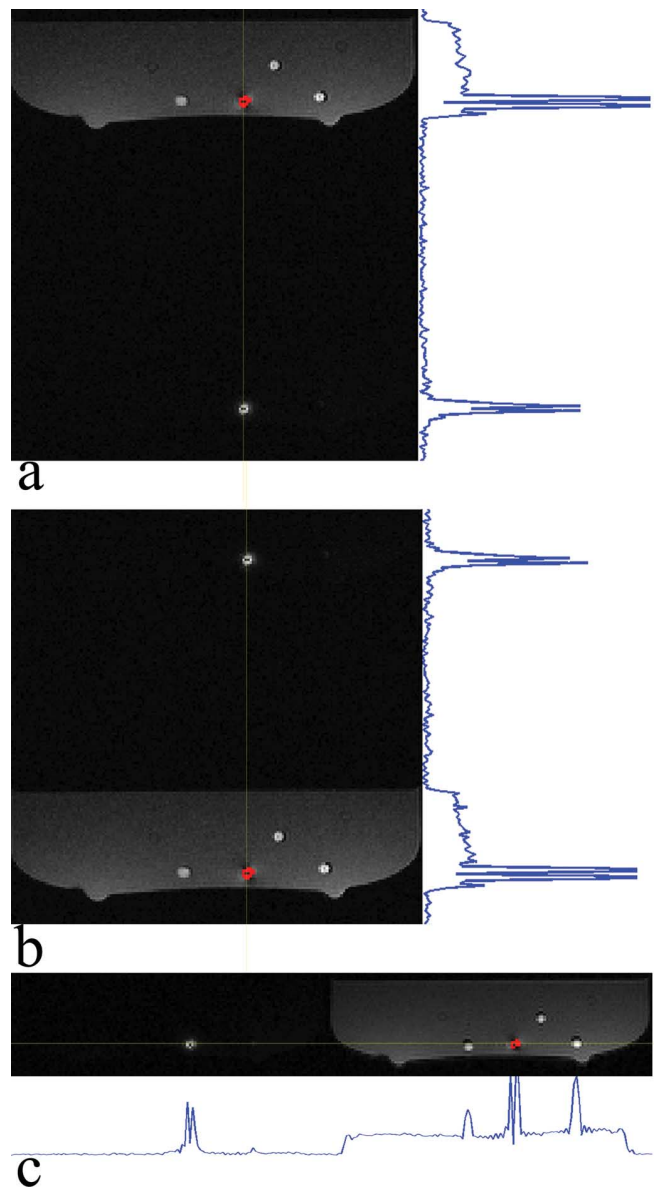

Fig. 8. Transversal images of the oil phantom, $\mathrm{KCl}$-solution-filled straws, and ICRF coil; the normalized performance plots through the yellow line, where $P=4$. a: The polarization vector circulates counter-clockwise and the phaseencoding direction is A-P. b: The polarization vector circulates clockwise, with the same phase-encoding direction as a. c: The polarization vector circulates counter-clockwise and the phase-encoding direction is L-R. All images show that suppressing the phantom and $\mathrm{KCl}$-filled straw signals are effective for $\mathrm{MR}$ guidance.
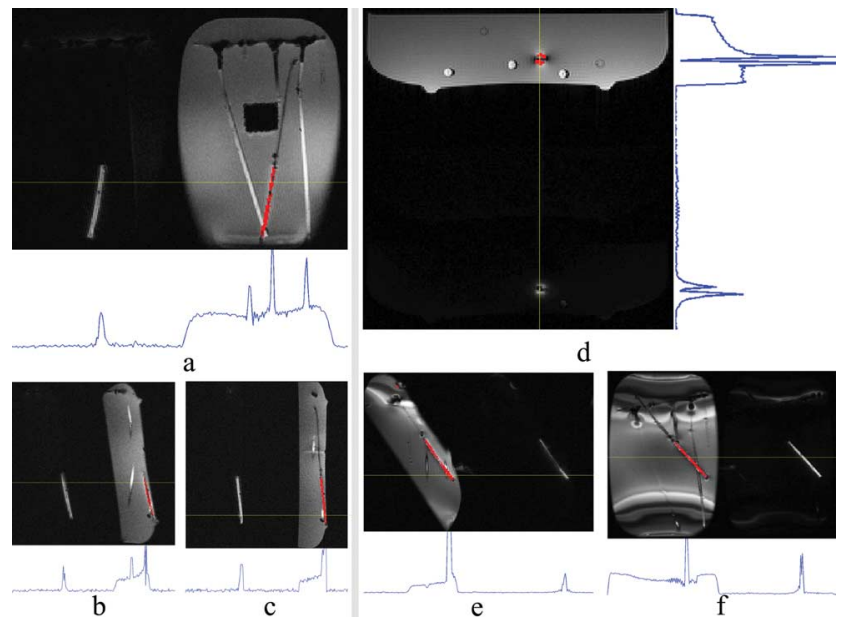

Fig. 9. Color-coded images of different planes when $P=4$, and performance plots with FLASH and TrueFISP sequences. (a) Coronal image, (b) oblique image and (c) sagittal image with FLASH. (d) Transversal image, (e) oblique image, and (f) coronal image with TrueFISP. Note that higher-order shimming is necessary for the coronal image.

quirement of the doubled FOV can be reduced by using different imaging planes. 


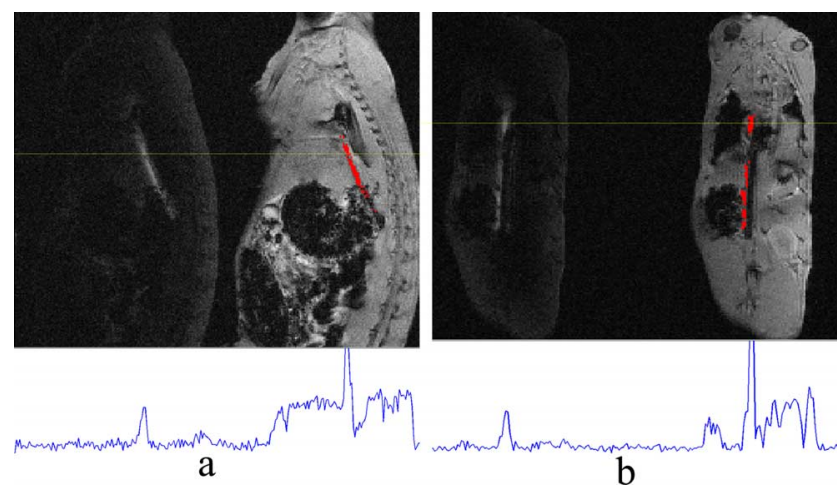

Fig. 10. Color-coded images and performance plots of the animal experiment. The catheter was inserted into the esophagus of the rabbit. (a) Sagittal image and (b) oblique image.

\section{Discussion}

The field created by the ICRF coil depends on the direction of the linearly polarized transmit field (Fig. 2). This fact is the main principle of the method. In the anatomy image case, only phase modulation occurs, which causes the shift of the anatomy image. However, as the phase of the RF excitation field changes, not only the phase but also the amplitude of the created ICRF coil field varies. Furthermore, the phase and magnitude modulation mechanisms occur differently than in the anatomy case. Modulations in k-space can be calculated analytically or by a computer simulation. Similar modulations of the phase and magnitude occur when a metallic wire is used instead of an ICRF coil, because the current induced on the wire is also dependent on the phase of the electrical field [36]. As the phase of the excitation changes, the phase of the electrical field and the phase of the wire current also change, and as a result, the field profile in the vicinity of the wire changes. This disturbance affects the k-space lines and a shifting, as in the ICRF coil case, occurs.

In this novel method, the anatomy image and the ghost shift in opposite directions, and therefore, a larger FOV and a longer scan time are necessary to avoid overlapping. This disadvantage can be eliminated using fast imaging methods and sequences. In addition, the shift direction of the ghost can be changed by switching the phase-encoding direction (Fig. 8), so if the appropriate phase encoding direction is chosen, the scan time increase can be reduced.

As stated above, the forward-polarized excitation has a better SAR performance than the linear excitation. The linear polarization used in this study (Fig. 1) doubles the average (whole body) SAR and quadruples the peak (maximum local) SAR. However, this is not the case for the circulating linear RF excitation. While the average SAR created by the circulating linear RF excitation is still twice that of the forward excitation, the peak SAR of this circulating excitation is reduced compared to the linear excitation because the electrical field of the linear excitation is not symmetric; therefore the location of the maximum local SAR varies from TR to TR. It should be noted that the increased SAR comes with the benefit of tracking the position and the rotational orientation of the catheter with an ICRF coil.
One may also compare this method to catheter tracking using reverse-polarized RF excitation [23]. Although the SAR (both the peak and the average) in a reverse-polarized excitation is half of the present method, the present method provides a higher SNR compared to the reverse-polarization method.

The proposed method requires a transmit array system to create circulating RF excitation. Although commercial transmit array systems have been introduced, they are still used for research only. General usage of the transmit array system includes homogenizing high magnetic field strengths, and the proposed application is promising for clinical usage.

To facilitate the circulating RF excitation, an ICRF coil must be attached to the catheter. In addition to the global SAR problem mentioned above, local heating due to the ICRF coil must be considered. In the authors' previous paper, the safety of the ICRF coils was analyzed rigorously [32]. Furthermore, the ICRF coil makes the catheter stiffer, which may make it more difficult to direct, thus the design of the catheter requires more attention.

There are three significant parameters to be manipulated depending on the application and imaging plane: $P$, the vector circulation direction, and the phase-encoding direction. In Fig. 7(a), $P$ was 16 , therefore the ICRF and the ghost were separated by 32 pixels, which successfully separated the ICRF coil image (yellow arrow) from the phantom in all cases. In Fig. 7(b), $P$ was 8 , and images were separated by 64 pixels. As an example of the second parameter, Fig. 8(a) shows a color-coded image of the counter-clockwise circulation of the vector with $P=4$. Shifting the circulation direction to clockwise swaps the anatomy image and the ghost [Fig. 8(b)]. The phase-encoding direction (the third parameter) is A-P in Fig. 8(a) and (b); changing it to L-R [Fig. 8(c)] changes the whole image.

At the site, the $\mathrm{x}$ - and $\mathrm{y}$-channels of the body coil were unbalanced; therefore, the transmit channels had to be adjusted to obtain linear excitations [23]. Because of this imperfection, residual anatomy images remained in the animal and phantom experiments. In this study, the system imperfection was not analyzed using quantitative measures, but both the phantom and animal images showed that the proposed system successfully obtained catheter information.

Symmetry of the ICRF coil in the $\phi$ direction of the cylindrical coordinates is a minor problem of the method. Rotational orientation is $\pi$ symmetric and the method cannot discriminate $\theta^{\circ}$ from $\theta^{\circ}+180^{\circ}$. This problem can be solved by using asymmetric ICRF coil designs.

The rotational orientation of a catheter is crucial information for certain applications, such as MR-guided intravascular focused ultrasound, side-looking optical imaging, and asymmetric needle puncturing, because devices or tools are placed on one side of the catheter. Therefore, targeting may require not only translating but also rotating the catheter. The theory of rotational orientation is derived in detail in the Appendix and the proof of principle is demonstrated by the phantom experiments. However, these were unable to be shown in the animal experiment, and a proper experimental setup must thus be designed to measure quantitative errors of rotational orientation results in the animal model. 


\section{CONCLUSION}

This study presented a novel method to simultaneously acquire the rotational orientation of and track an ICRF coil built on a catheter. The anatomy and ICRF coil images are separated, then the orientation is calculated and a color-coded image of the ICRF coil is reconstructed for tracking. Phantom experiments demonstrated the method's proof of principle using both FLASH and TrueFISP sequences.

\section{APPENDIX}

\section{Small-Tip Angle Approximation}

Validity of the method for a larger flip angle is shown with a computer simulation (Fig. 3) and the general theory of the present study is given in the Method section. A small-tip angle approximation can be used for a more detailed formulation and analysis. Assume $M_{T, \text { Anatomy }}$ is the spin magnetization of the anatomy and that the signal resulting from a conventional sequence (Fig. 1) is

$$
S_{\text {Anatomy }}(t)=\iint\left(M_{T, \text { Anatomy }}\right) e^{-i 2 \pi k_{x} x} e^{-i 2 \pi k_{y} y} d x d y
$$

where $k_{x}$ and $k_{y}$ are spatial frequency variables in the $x$ and $y$ directions, respectively. In this signal equation, assuming $P=$ 4 , a generalized magnetization after the $p$ th RF (or $p$ th TR) can be expressed as

$$
M_{p T, \text { Anatomy }}^{+}(t)=M_{T, \text { Anatomy }} e^{i p \pi / 2} \text {. }
$$

Assuming $y$ is the phase-encoding direction, $p$ can be expressed in terms of imaging parameters as

$$
p=k_{y} N_{y} \Delta y .
$$

Above, $\Delta y$ is the image resolution in the phase-encoding direction. Substituting (5) into (3) gives

$$
\begin{array}{r}
S_{\text {Anatomy }}(t)=\iint\left(M_{T, \text { Anatomy }} e^{-i 2 \pi k_{y}\left(y-N_{y} \Delta y / 4\right)}\right) \\
\times e^{-i 2 \pi k_{x} x} d x d y .
\end{array}
$$

Equation (6) states that the modified RF excitation causes a shift in the image location in the phase-encoding direction with an amount of $N_{y} / P$, where $P=4$.

As stated above in the general theory of the method, the effect of changing the linear polarization vector to the ICRF coil is more complex than the anatomy. The transmit field induces a current on the ICRF coil and the induced current creates a secondary magnetic field. This time-dependent magnetic field created by the ICRF coil can be represented as

$$
\vec{B}_{\mathrm{ICRF}, t}(t)=I_{\mathrm{ICRF}, t}(t) \vec{B}_{\mathrm{ICRF}}^{\prime}
$$

where $I_{\mathrm{ICRF}, t}(t)$ is the time-dependent current due to the transmit magnetic field and is expressed as

$$
I_{\mathrm{ICRF}, t}(t)=B_{t}(t) \cos \left(\omega_{o} t+\sigma\right) \widehat{a}_{t} \cdot \vec{s} .
$$

Above, $B_{t}(t)$ is the peak value of the time-dependent transmit magnetic field envelope function, $\omega_{o}$ is the Larmor frequency, $\sigma$ and $\widehat{a}_{t}$ are the phase and unit vectors of $B_{t}(t)$, and $\vec{s}$ is the surface normal vector of the ICRF coil, given by

$$
\vec{s}=s\left(\cos \theta \widehat{a}_{x}+\sin \theta \widehat{a}_{y}\right)
$$

where $s$ is the area of the ICRF coil. For every different RF excitation mentioned in Fig. 1, the dot product of the surface normal vector of the ICRF coil and the unit vector of the transmit magnetic field in (8) causes different complex parameters.

$\operatorname{In}(7), \vec{B}_{\text {ICRF }}^{\prime}$ is the peak value of the magnetic field generated by the ICRF coil at a point of interest when a unit current is applied to an imaginary terminal of the coil

$$
\vec{B}_{\mathrm{ICRF}}^{\prime}=B_{c o}\left(\cos (\theta+\eta) \widehat{a}_{x}+\sin (\theta+\eta) \widehat{a}_{y}\right) .
$$

Above, $B_{c o}$ is the magnitude of the magnetic field, $\theta+\eta$ is the orientation of the magnetic field vector at the point of interest, $\eta$ is the phase term of the magnetic field related to other than the orientation of the ICRF coil. Note that the field vector orientation is directly related to the orientation of the ICRF coil, therefore $\theta$ appears in the magnetic field expression. Using the above equations, the $x$ and $y$ components of the $\vec{B}_{\mathrm{ICRF}, t}(t)$ can be expressed as

$$
\begin{aligned}
B_{\mathrm{ICRF}, t x}(t)= & B_{t}(t) B_{c o} s\left[\begin{array}{lll}
\widehat{a}_{t} \cdot(\cos \theta & \widehat{a}_{x}+\sin \theta & \widehat{a}_{y}
\end{array}\right] \\
& \cdot \cos \left(\omega_{o} t+\sigma\right) \cos (\theta+\eta) \\
B_{\mathrm{ICRF}, t y}(t)= & B_{t}(t) B_{c o} s\left[\begin{array}{lll}
\widehat{a}_{t} \cdot(\cos \theta & \widehat{a}_{x}+\sin \theta & \widehat{a}_{y}
\end{array}\right] \\
& \cdot \cos \left(\omega_{o} t+\sigma\right) \sin (\theta+\eta) .
\end{aligned}
$$

Converting the lab frame expressions into the rotating frame will be useful for calculations [37]

$$
\begin{aligned}
& B_{x}^{+}(t)=B_{\mathrm{ICRF}, t x}(t) \cos \left(\omega_{o} t\right)+B_{\mathrm{ICRF}, t y}(t) \sin \left(\omega_{o} t\right) \\
& B_{y}^{+}(t)=-B_{\mathrm{ICRF}, t x}(t) \sin \left(\omega_{o} t\right)+B_{\mathrm{ICRF}, t y}(t) \cos \left(\omega_{o} t\right)
\end{aligned}
$$

where $B_{x}^{+}(t)$ and $B_{y}^{+}(t)$ are the $x$ and $y$ components of the positively rotating frame. Ignoring $2 \omega_{o} t$ components, which are not effective in NMR

$$
\begin{aligned}
& B_{x}^{+}(t)=-B \cos (\theta+\eta) \sin \sigma+B \sin (\theta+\eta) \cos \sigma \\
& B_{y}^{+}(t)=-B \cos (\theta+\eta) \cos \sigma-B \sin (\theta+\eta) \sin \sigma .
\end{aligned}
$$

Above, $B=B_{t}(t) B_{c o} s\left[\widehat{a}_{t} \cdot\left(\cos \theta \widehat{a}_{x}+\sin \theta \widehat{a}_{y}\right)\right] / 2$.

These rotating frame components are enough to express magnetizations. For the sake of simplicity, assume a small-tip angle to obtain the $x$ and $y$ components of the magnetization

$$
\begin{aligned}
& d M_{x}^{+}(t) / d t \approx-\gamma M_{o} B_{y}^{+}(t) \\
& d M_{y}^{+}(t) / d t \approx \gamma M_{o} B_{x}^{+}(t) .
\end{aligned}
$$

Here, $\gamma$ is the gyromagnetic ratio and $M_{o}$ is the instantaneous magnetic moment per sample voxel immediately after applying a $90^{\circ}$ pulse. Assuming a rectangular pulse

$$
\begin{aligned}
& M_{x}^{+}(t)=-\gamma \tau M_{o} B_{y}^{+}(t) \\
& M_{y}^{+}(t)=\gamma \tau M_{o} B_{x}^{+}(t) .
\end{aligned}
$$


Above, $\tau$ is the duration of the pulse. The complex transverse magnetization of the ICRF coil magnetic field can be written as $M_{T, \mathrm{ICRF}}^{+}(t)=M_{x}^{+}(t)+i M_{y}^{+}(t)$, and substituting (15) gives

$$
M_{T, \mathrm{ICRF}}^{+}(t)=M_{T, \mathrm{ICRF}} e^{i \theta}\left[\widehat{a}_{t} \cdot\left(\cos \theta \widehat{a}_{x}+\sin \theta \widehat{a}_{y}\right)\right]
$$

where $M_{T \text {.ICRF }}=\gamma \tau M_{o} e^{i \eta} e^{i \sigma} B_{t}(t) B_{c o} s / 2$. The remaining terms on the right side of (16) are directly related to the orientation of the ICRF coil. As the RF pulse scheme changes, the complex transverse magnetization of the ICRF coil changes, similar to the anatomy case. However, the orientation-related complex term in (16) has a very important difference. At the first TR, the unit vector of the transmit magnetic field will be $\widehat{a}_{t}=\widehat{a}_{x}$, because its direction shows the $x$-axis. Substituting this information into (16) results in the first complex transverse magnetization of the ICRF coil

$$
M_{0 T, \mathrm{ICRF}}^{+}(t)=M_{T, \mathrm{ICRF}} e^{i \theta} \cos \theta .
$$

The second, third, and fourth transmit magnetic field unit vectors become $\widehat{a}_{y},-\widehat{a}_{x}$, and $-\widehat{a}_{y}$, respectively, and one can generalize the $p$ th $\mathrm{RF}$ as

$$
M_{p T, \mathrm{ICRF}}^{+}(t)=M_{T, \mathrm{ICRF}}\left(e^{i 2 \theta} e^{i p \pi / 2}+e^{-i p \pi / 2}\right) / 2 .
$$

The above equation states that the modified RF pulse scheme given in Fig. 1 adds a phase term $\left(e^{i 2 \theta} e^{i p \pi / 2}+e^{-i p \pi / 2}\right) / 2$ to the ICRF signal. Also, not only does the phase change in each TR, but so does the magnitude of the effective RF field.

For the case explained above, the signal expression can be given as

$$
\begin{array}{r}
S_{\mathrm{ICRF}}(t)=\iint\left(M_{T, \mathrm{ICRF}}\left(e^{i 2 \theta} e^{i p \pi / 2}+e^{-i p \pi / 2}\right)\right) \\
\times e^{-i 2 \pi\left(k_{x} x+k_{y} y\right)} d x d y / 2 .
\end{array}
$$

Substituting (5) into (19) gives

$$
\begin{aligned}
S_{\mathrm{ICRF}}(t)= & \iint M_{T, \mathrm{ICRF}} \\
& \times e^{-i 2 \pi k_{y}\left(y-N_{y} \Delta y / 4\right)} e^{-i 2 \pi k_{x} x} d x d y / 2 . \\
& +\iint\left(M_{T, \mathrm{ICRF}} e^{i 2 \theta}\right) \\
& \times e^{-i 2 \pi k_{y}\left(y+N_{y} \Delta y / 4\right)} e^{-i 2 \pi k_{x} x} d x d y / 2 .
\end{aligned}
$$

One can derive two conclusions from (20). First, the modified RF scheme causes shifting of the original image. The anatomy shifts in the phase-encoding direction by $N_{y} / 4$ pixels; the original ICRF coil image also shifts in the phase-encoding direction in alignment with the anatomy, and the ghost shifts in the reverse direction. These shifts enable the separation of the ICRF coil image from the anatomy image, so that the ICRF coil image can be color-coded and placed into the anatomy image and the catheter can be tracked. Next, (20) states that the phase difference of the ICRF coil and the ghost image is equal to two times the rotational orientation angle of the ICRF coil, $2 \theta$.

Assume $y_{o}$ is the central line between the ICRF coil image and the ghost. Although the exact location of the original image is not known, $y_{o}$ is related to the total number of phase lines and number of pulses in one cycle and is given by $y_{o}=N_{y}-$ $2 N_{y} / P$. As a result, for the particular case where $P=4, y_{o}$ equals the center row of the image $N_{y} / 2$. In a more general formula for an arbitrary $P$, the anatomy and ICRF coil signals can be expressed as

$$
\begin{aligned}
S_{\text {Anatomy }}(t)= & \iint M_{T, \text { Anatomy }} e^{-i 2 \pi k_{y}\left(y-N_{y} \Delta y / P\right)} \\
& \times e^{-i 2 \pi k_{x} x} d x d y \\
S_{\text {ICRF }}(t)= & \iint M_{T, \mathrm{ICRF}} e^{-i 2 \pi k_{y}\left(y-N_{y} \Delta y / P\right)} \\
& \times e^{-i 2 \pi k_{x} x} d x d y / 2 \\
& +\iint\left(M_{T, \mathrm{ICRF}} e^{i 2 \theta}\right) e^{-i 2 \pi k_{y}\left(y+N_{y} \Delta y / P\right)} \\
& \times e^{-i 2 \pi k_{x} x} d x d y / 2 .
\end{aligned}
$$

Equation (21) states that the resulting image shift is $N_{y} / P$ pixels; therefore the shift can be adjusted by changing the number of cycles over the phase-encoding direction in k-space. For example, the amount of shift can be increased by decreasing the value of $P$ and vice versa while the direction of the shift can be changed by changing the sign of the modulation.

\section{ACKNOWLEDGMENT}

The authors would like to thank R. Nelson for her valuable editorial support.

\section{REFERENCES}

[1] O. Ocali and E. Atalar, "Intravascular magnetic resonance imaging using a loopless catheter antenna," Magn. Reson. Med., vol. 37, pp. 112-118, Jan. 1997

[2] C. L. Dumoulin, S. P. Souza, and R. D. Darrow, "Real-time position monitoring of invasive devices using magnetic resonance," Magn. Reson. Med., vol. 29, pp. 411-415, Mar. 1993.

[3] V. D. Kochli, G. C. McKinnon, E. Hofmann, and G. K. Vonschulthess, "Vascular interventions guided by ultrafast MR-imaging_Evaluation of different materials," Magn. Reson. Med., vol. 31, pp. 309-314, Mar. 1994.

[4] K. Ratnayaka, A. Z. Faranesh, M. A. Guttman, O. Kocaturk, C. E. Saikus, and R. J. Lederman, "Interventional cardiovascular magnetic resonance: Still tantalizing," J. Cardiovasc. Magn. Reson., vol. 10, p. 62, 2008.

[5] O. Unal, J. Li, W. Cheng, H. Yu, and C. M. Strother, "MR-visible coatings for endovascular device visualization," J. Magn. Reson. Imag., vol. 23, pp. 763-769, May 2006.

[6] H. H. Quick, M. O. Zenge, H. Kuehl, G. Kaiser, S. Aker, S. Massing, S. Bosk, and M. E. Ladd, "Interventional magnetic resonance angiography with no strings attached: Wireless active catheter visualization," Magn. Reson. Med., vol. 53, pp. 446-455, Feb. 2005.

[7] P. A. Bottomley, A. Kumar, W. A. Edelstein, J. M. Allen, and P. V. Karmarkar, "Designing passive MRI-safe implantable conducting leads with electrodes," Med. Phys., vol. 37, pp. 3828-3843, Jul. 2010.

[8] S. Weiss, T. Kuehne, F. Brinkert, G. Krombach, M. Katoh, T. Schaeffter, R. W. Guenther, and A. Buecker, "In vivo safe catheter visualization and slice tracking using an optically detunable resonant marker," Magn. Reson. Med., vol. 52, pp. 860-868, Oct. 2004.

[9] C. E. Saikus, K. Ratnayaka, I. M. Barbash, J. H. Colyer, O. Kocaturk, A. Z. Faranesh, and R. J. Lederman, "MRI-guided vascular access with an active visualization needle," J. Magn. Reson. Imag., vol. 34, pp. 1159-1166, Nov. 2011.

[10] O. Kocaturk, C. E. Saikus, M. A. Guttman, A. Z. Faranesh, K. Ratnayaka, C. Ozturk, E. R. McVeigh, and R. J. Lederman, "Whole shaft visibility and mechanical performance for active MR catheters using copper-nitinol braided polymer tubes," J. Cardiovasc Magn. Reson., vol. 11, p. 29, 2009. 
[11] O. Kocaturk, A. H. Kim, C. E. Saikus, M. A. Guttman, A. Z. Faranesh, C. Ozturk, and R. J. Lederman, ““'Active two-channel 0.035” guidewire for interventional cardiovascular MRI," J. Magn. Reson. Imag., vol. 30, pp. 461-465, Aug. 2009.

[12] S. Weiss, D. Wirtz, B. David, S. Krueger, O. Lips, D. Caulfield, S. F. Pedersen, J. Bostock, R. Razavi, and T. Schaeffter, "In vivo evaluation and proof of radiofrequency safety of a novel diagnostic MR-electrophysiology catheter," Magn. Reson. Med., vol. 65, pp. 770-777, Mar. 2011.

[13] K. N. Kurpad and O. Unal, "Multimode intravascular RF coil for MRIguided interventions," J. Magn. Reson. Imag., vol. 33, pp. 995-1002, Apr. 2011

[14] M. Bock, S. Muller, S. Zuehlsdorff, P. Speier, C. Fink, P. Hallscheidt, R. Umathum, and W. Semmler, "Active catheter tracking using parallel MRI and real-time image reconstruction," Magn. Reson. Med., vol. 55, pp. 1454-1459, Jun. 2006.

[15] A. K. Homagk, R. Umathum, M. Korn, M. A. Weber, P. Hallscheidt, W. Semmler, and M. Bock, "An expandable catheter loop coil for intravascular MRI in larger blood vessels," Magn. Reson. Med., vol. 63, pp. 517-523, Feb. 2010.

[16] K. J. Anderson, G. Leung, A. J. Dick, and G. A. Wright, "Forwardlooking intravascular orthogonal-solenoid coil for imaging and guidance in occlusive arterial disease," Magn. Reson. Med., vol. 60, pp. 489-495, Aug. 2008.

[17] J. H. Hwang, Y. Zhou, C. Warren, A. A. Brayman, and L. A. Crum, "Targeted venous occlusion using pulsed high-intensity focused ultrasound," IEEE Trans. Biomed. Eng., vol. 57, no. 1, pp. 37-40, Jan. 2010.

[18] C. Di Mario, G. Gorge, R. Peters, P. Kearney, F. Pinto, D. Hausmann, C. von Birgelen, A. Colombo, H. Mudra, J. Roelandt, and R. Erbel, "Clinical application and image interpretation in intracoronary ultrasound. Study group on intracoronary imaging of the working group of coronary circulation and of the subgroup on intravascular ultrasound of the working group of echocardiography of the european society of cardiology," Eur. Heart J., vol. 19, pp. 207-229, Feb. 1998.

[19] M. P. Fronheiser, E. D. Light, and S. W. Smith, "Real-time 3D ultrasound with multiple transducer arrays," in Proc. IEEE Ultrason. Symp. 2004, vol. 1, pp. 786-789.

[20] K. J. Anderson, A. J. Dick, and G. A. Wright, "Catheter tracking with phase information," presented at the ISMRM, Kyoto, Japan, 2004

[21] K. J. Anderson, G. C. Scott, and G. A. Wright, "Catheter tracking with phase information in a magnetic resonance scanner," IEEE Trans. Med. Imag., vol. 31, no. 6, pp. 1173-1180, Jun. 2012.

[22] G. Adriany, P. F. Van de Moortele, F. Wiesinger, S. Moeller, J. P. Strupp, P. Andersen, C. Snyder, X. Zhang, W. Chen, K. P. Pruessmann, P. Boesiger, T. Vaughan, and K. Uğurbil, "Transmit and receive transmission line arrays for 7 Tesla parallel imaging," Magn. Reson. Med., vol. 53 , pp. $434-445,2005$.
[23] H. Celik and E. Atalar, "Reverse polarized inductive coupling to transmit and receive radiofrequency coil arrays," Magn. Reson. Med., vol. 67, pp. 446-456, 2012.

[24] U. Katscher, P. Börnert, C. Leussler, and J. S. van den Brink, "Transmit SENSE," Magn. Reson. Med., vol. 49, pp. 144-150, 2003.

[25] R. G. Pinkerton, J. P. Near, E. A. Barberi, R. S. Menon, and R. Bartha, "Transceive surface coil array for MRI of the human prostate at 4T," Magn. Reson. Med., vol. 57, pp. 455-458, 2007.

[26] H. Celik, A. Ulutürk, Y. Eryaman, T. Tal1, and E. Atalar, "A novel catheter tracking method using reversed polarization," presented at the 14th Annu. Meet. ISMRM, Seattle, WA, 2006.

[27] H. Quick, M. Zenge, H. Kuehl, G. Kaiser, S. Aker, H. Eggebrecht, S. Massing, and M. Ladd, "Wireless active catheter visualization: Passive decoupling methods and their impact on catheter visibility," presented at the 13th Annu. Meet. ISMRM, Miami Beach, FL, 2005.

[28] H. H. Quick, H. Kuehl, G. Kaiser, S. Bosk, J. F. Debatin, and M. E. Ladd, "Inductively coupled stent antennas in MRI," Magn. Reson. Med., vol. 48, pp. 781-790, Nov. 2002

[29] H. H. Quick, H. Kuehl, G. Kaiser, D. Hornscheidt, K. P. Mikolajczyk, S. Aker, J. F. Debatin, and M. E. Ladd, "Interventional MRA using actively visualized catheters, TrueFISP, and real-time image fusion," Magn. Reson. Med., vol. 49, pp. 129-137, Jan. 2003.

[30] M. Bernstein, K. King, and X. Zhou, Handbook of MRI Pulse Sequences. New York: Academic, 2004, p. 420.

[31] F. Schick, J. Forster, J. Machann, P. Huppert, and C. D. Claussen, "Highly selective water and fat imaging applying multislice sequences without sensitivity to B1 field inhomogeneities," Magn. Reson. Med., vol. 38, pp. 269-274, Aug. 1997.

[32] H. Celik, A. Uluturk, T. Tali, and E. Atalar, "A catheter tracking method using reverse polarization for MR-guided interventions," Magn. Reson. Med., vol. 58, pp. 1224-1231, Dec. 2007.

[33] C. E. Hayes, W. A. Edelstein, J. F. Schenck, O. M. Mueller, and M. Eash, "An efficient, highly homogenous radiofrequency coil for wholebody NMR imaging at 1.5 T," J. Magn. Reson., vol. 63, pp. 622-628, 1985.

[34] A. V. Oppenheim, A. S. Willsky, and S. H. Nawab, Signals \& Systems, 2nd ed. Upper Saddle River, NJ: Prentice-Hall, 1996.

[35] M. A. Bernstein, M. Grgic, T. J. Brosnan, and N. J. Pelc, "Reconstructions of phase contrast, phased array multicoil data," Magn. Reson. Med., vol. 32, pp. 330-334, Sept. 1994.

[36] V. Acikel and E. Atalar, "Modeling of RF induced implant lead current for MRI," presented at the 17th Annu. Meet. ISMRM, Honolulu, HI, 2009.

[37] D. I. Hoult, "The principle of reciprocity in signal strength calculations-A mathematical guide," Concepts Magn. Reson., vol. 12, pp. 173-187, 2000. 